\title{
Infrared Observations of the Asymmetric Mass Loss of an AGB Star
}

\author{
Foteini Lykou ${ }^{1,2, *}$ (D), Albert A. Zijlstra ${ }^{3}$ (D) Jacques Kluska ${ }^{4,5}$ (D) Eric Lagadec $^{6}$ (D), \\ Peter G. Tuthill ${ }^{7}$, Adam Avison ${ }^{3}$, Barnaby R. M. Norris ${ }^{7}$ and Quentin A. Parker ${ }^{1,2}$ (D) \\ 1 Department of Physics, The University of Hong Kong, Pokfulam Road, Hong Kong, China; \\ quentinp@hku.hk \\ 2 Laboratory for Space Research, The University of Hong Kong, 100 Cyberport Road, Cyberport, \\ Hong Kong, China \\ 3 Jodrell Bank Centre for Astrophysics, The University of Manchester, Oxford Road, Manchester M13 9PL, UK; \\ albert.zijlstra@manchester.ac.uk (A.A.Z.); adam.avison@manchester.ac.uk (A.A.) \\ 4 Institute for Astronomy, KU Leuven, Celestijnenlaan 200D B2401, 3001 Leuven, Belgium; \\ jacques.kluska@kuleuven.be \\ 5 School of Physics, University of Exeter, Stocker Road, Exeter EX4 4QL, UK \\ 6 Observatoire de la Côte d'Azur, Laboratoire Lagrange, Université Côte d'Azur, 06304 Nice, France; \\ eric.lagadec@oca.eu \\ 7 Sydney Institute of Astronomy, School of Physics, The University of Sydney, \\ Camperdown, NSW 2006, Australia; peter.tuthill@sydney.edu.au (P.G.T.); \\ barnaby.norris@sydney.edu.au (B.R.M.N.) \\ * Correspondence: lykoufc@hku.hk
}

Received: 31 July 2018; Accepted: 9 October 2018; Published: 12 October 2018

\begin{abstract}
We report on the observations of the circumstellar envelope of the AGB star II Lup in the near- and mid-infrared with the use of direct imaging and interferometric techniques. Our findings indicate that the circumstellar envelope is not spherically symmetric and that the majority of the emission originates within 0.5 arcsec from the star.
\end{abstract}

Keywords: infrared interferometry; AGB stars; stellar evolution; observations; aperture masking

\section{Introduction}

The study of the asymmetries found in the majority of planetary nebulae have been the core subject of this conference series. However, as it has been stressed in the last two APNmeetings, these asymmetries ought to be generated by mechanisms that act as early as the AGB phase. It is currently believed that these mechanisms are the result of an interplay between two shaping mechanisms, binarity and magnetic fields, and both could be investigated in AGB stars.

Different spatial scales at different wavelength ranges help us to dissect different parts of the circumstellar envelope (CSE) of evolved stars. The outer and colder layers of a CSE are observed in the sub-mm and far-infrared wavelengths (e.g., 1" with ALMA and Herschel telescopes), while the warmer layers of the CSE can be observed in the mid-infrared (e.g., 0.5" with VISIR/Very Large Telescope (VLT)). The layers of the CSE closer to the central star, including the stellar photosphere and its hot, dusty atmosphere, can be explored in the near-infrared (e.g., $\leq 0.2^{\prime \prime}$ with NACO/VLT).

Until now, there have been only a few imaging surveys that have explored this: two in the sub-mm wavelengths [1,2], one in the far-infrared [3] and one in the mid-infrared [4]. The initial target lists are very similar in all four surveys, and the majority of the objects were found to depart from spherical symmetry at large spatial scales $\left(\geq 1^{\prime \prime}\right)$. However, many of the targets, especially the inner layers of the CSEs of the AGB stars, were unresolved at smaller spatial scales $\left(\leq 0.4^{\prime \prime}\right)$. We therefore initiated a survey 
of 22 objects in the period 2009-2018 to look for any asymmetries in evolved stars and explored the possibility of binary interactions with the use of interferometry, to access the sub-arcsecond angular scales needed, and direct imaging in the infrared. The targets were selected from the initial list of [4]. Some of the most striking results of this survey have been presented in [5-7], and the analysis of the final sample is on-going.

One of the AGB stars in our sample is II Lup. It is a carbon-rich AGB star, and the typical masses for such stars range from 1-4 $\mathrm{M}_{\odot}$. The star is placed near the tip of the AGB $\left(m_{\mathrm{bol}}=3.73,[8]\right)$ in the evolutionary tracks of [9], and therefore, it is not yet hot enough to ionize its CSE. II Lup shows a peculiar variability in the near-infrared ( $J-L$ bands), where its light curve can be fitted by two periods: a short-term one at 575 days and a long-term one at $\sim 19$ years [10]. The latter was characterized as an obscuration event and is thought to be related to asymmetric ${ }^{1}$ mass loss [10]. We reported the first-ever detection of asymmetries in II Lup's CSE in the near-infrared and sub-mm wavelengths in Lykou et al. [11]. Here, we present complementary (near- and mid-infrared) images to that work.

\section{Results}

The observations of II Lup in the near- and mid-infrared were carried with $8 \mathrm{~m}$-class telescopes by Lykou et al. [11]. A brief description of the observing modes is given below, while the results can be easily compared to [11].

II Lup was observed with the VISIR mid-infrared instrument in March 2016 (JD = 2,457,468). VISIR is a spectrometer and imager on the Very Large Telescope (VLT) [12]. The observations were carried in burst mode, which can provide diffraction-limited images (e.g., $\theta_{\text {res }}=0.25^{\prime \prime}$ at $8 \mu \mathrm{m}$ ). Observations of the science target and a calibrator were obtained with the PAH_1 and PAH_2 filters ${ }^{2}$ (hereby, $8 \mu \mathrm{m}$ and $11 \mu \mathrm{m}$ for simplicity). The data were reduced and analysed using the method of [4]. The science data suffered from saturation from the central star; therefore, we present here a tentative analysis of this dataset.

Each science image was deconvolved following the Lucy-Richardson method, using the cropped images of the corresponding PSF calibrator (radius $\sim 1$ arcsec) and thus removing the noise of the otherwise empty field-of-view $\left(10^{\prime \prime} \times 10^{\prime \prime}\right)$. This significantly minimizes the computation time for the deconvolution. The process was stopped after 30 iterations. Each image was then smoothed (convolved) with a two-pixel Gaussian kernel. The deconvolved images $\left(2.9^{\prime \prime} \times 2.9^{\prime \prime}\right)$ are shown in Figure 1.

As expected, the central star is unresolved, and any deviations from symmetry within the resolution elements in Figure 1 should be ignored. The morphology of the envelope at $8 \mu \mathrm{m}$ is relatively similar to that at $L$ and $M$ (cf. Figure 7 in Lykou et al. [11]) with respect to its north-south orientation. At $11 \mu \mathrm{m}$, the CSE appears to be more round with a small displacement to the north; however, the brightness distribution is not entirely uniform. Although the data suffered from saturation, we can tentatively deduce that the CSE extends up to a radius of $0.47^{\prime \prime}$ and $0.6^{\prime \prime}$ at $8 \mu \mathrm{m}$ and $11 \mu \mathrm{m}$, respectively (black contours in Figure 1). Therefore, the CSE appears to be a relatively compact object in the mid-infrared with respect to the size of the envelope in the far-infrared (e.g., $70 \mu \mathrm{m}$ PACS/Herschel map where CSE size 40"; see also [13]). However, the size of the photosphere must be less than $0.25^{\prime \prime}$.

II Lup was also observed in June 2010 (JD $=2,455,377)$ in the near-infrared $(K, L$ and $M)$ with the Sparse Aperture Masking mode on NACO/VLT [14-17]. This technique uses a nine-hole mask that converts the single-dish $8 \mathrm{~m}$-class telescope into an interferometer with 36 baselines $^{3}$ to produce diffraction-limited images (e.g., $\theta_{\text {res }}=72$ mas in $M$ ). The data reduction used a custom-made pipeline, and the analysis and image reconstruction processes were performed as described in $[6,11,17]$.

\footnotetext{
The term "asymmetric" will hereby refer to any non-spherical symmetry.

PAH_1: $\lambda=8.54 \mu \mathrm{m}, \Delta \lambda=0.42 \mu \mathrm{m}$; PAH_2 : $\lambda=11.25 \mu \mathrm{m}, \Delta \lambda=0.59 \mu \mathrm{m})$.

The baseline range was $1.3-6.9 \mathrm{~m}$ for various azimuths.
} 

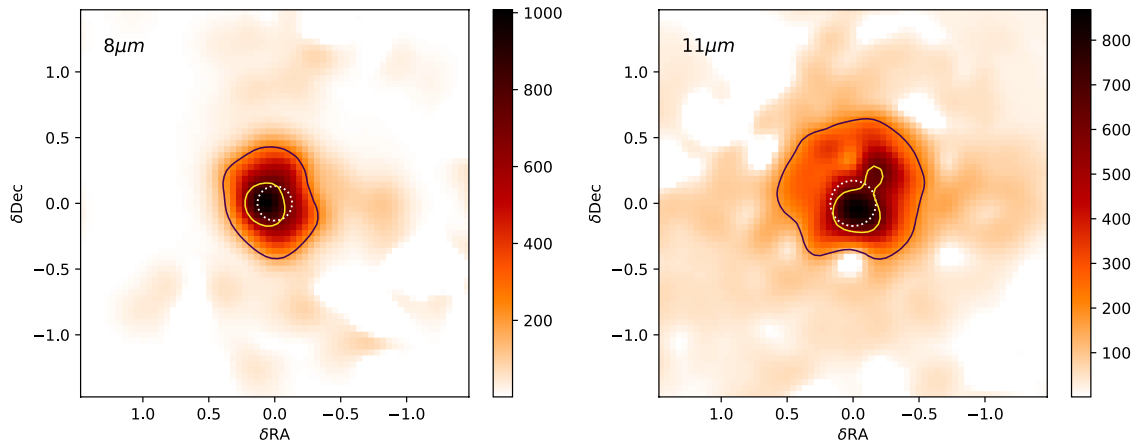

Figure 1. VISIR/Very Large Telescope (VLT) deconvolved images of II Lup at $8 \mu \mathrm{m}$ (left) and $11 \mu \mathrm{m}$ (right) in squared-root intensity scale. The resolution element is indicated by the dotted, white circles in the core of each image, while the black and yellow contours indicate the $5 \%$ and $50 \%$ levels of the peak intensity, respectively. North is up and east is to the left. The colour bars indicate a relative intensity scale.

Figure 2 shows the image reconstruction for the $M$ data with the MiRA algorithm $[18,19]$. It is evident that the circumstellar envelope departs from spherical symmetry and extends up to 120 milliarcseconds (mas) north with another protrusion extending approximately 80 mas south-west. The photosphere of the AGB star is unresolved, and therefore, its size must be smaller than 32 mas, as shown by the $K$-band images of Lykou et al. [11]. The entire structure fits well inside the resolution element (white circle) of the VISIR $8 \mu \mathrm{m}$ image (left panel, Figure 1); hence, we were able to resolve the CSE of this AGB star at the smallest spatial scale possible with this technique in the $M$ band.

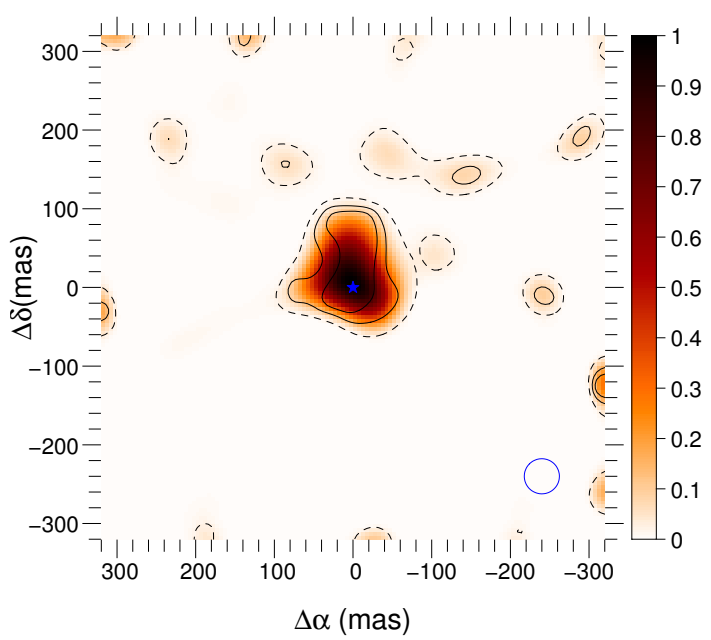

Figure 2. II Lup's M band image reconstructed with MiRA. The flux scale has been normalized to unity, and the contours represent the 5, 3 (solid) and 1 (dash) significance levels. The resolution element is indicated by a blue circle and the position of the central star by a blue asterisk.

The upper limits of the radii of CSE's detected layers are tabulated in Table 1. The angular sizes were converted to physical values for an adopted distance of $590 \mathrm{pc}$ [13]. Using these sizes and assuming that the expansion velocity of II Lup $\left(v_{\exp }=23 \mathrm{~km} \mathrm{~s}^{-1}\right.$; [20]) remains the same throughout the CSE, the approximate time scales for the expansion of the layers ought to be shorter than 14.6, 57,

4 There are no high-resolution, infrared, spectroscopic measurements for this star in the current literature. 
73 and 2430 years, respectively. This would suggest that the NACO observations detected a relatively recent mass-loss event.

Table 1. Upper-limits for the circumstellar envelope sizes for an adopted distance of 590 pc.

\begin{tabular}{cccc}
\hline \multirow{2}{*}{ Band } & \multicolumn{2}{c}{ Radius } & \multirow{2}{*}{ Reference } \\
\cline { 2 - 3 } & (arcsec) & (au) & \\
\hline$M$ & 0.12 & 71 & this work \\
$8 \mu \mathrm{m}$ & 0.47 & 277 & this work \\
$11 \mu \mathrm{m}$ & 0.6 & 354 & this work \\
$70 \mu \mathrm{m}$ & 20 & 11,800 & this work and [13] \\
\hline
\end{tabular}

\section{Discussion}

The near- and mid-infrared observations of II Lup reveal that the hot- and warm-dust layers of its circumstellar envelope are very compact (size $\leq 1.2^{\prime \prime}$ ) with respect to the larger and spherically-symmetric, cold-dust envelope (size $\sim 40 " ;[3,13])$. Although the mid-infrared images are not conclusive on the asymmetry of the circumstellar envelope, which was mainly due to the quality of the data and the imaging method used, the near-infrared data indicate an oblate envelope (Figure 2).

These images therefore indicate that the morphology of the dusty, circumstellar envelope of II Lup is not spherically symmetric, which confirms the hypothesis of [10] for this star, as well as the findings of [11]. The mechanism of these asymmetries could be the influence of a binary companion orbiting the AGB star, but no such star was found in spatial scales $\geq 0.2^{\prime \prime}$ (or else, orbital separations $\geq 118$ AU at the adopted distance of 590 pc). However, if such a companion exists closer to the AGB star, this hypothesis can only be tested with new interferometric observations, preferably made with larger scale interferometers such as the VLTI. The analysis of the current images suggests that we have detected layers of the CSE that were recently formed (age $\leq 80$ years). We expect that any planetary nebula created from this star in the future will be shaped by the same mechanism that created the asymmetries in the current circumstellar envelope.

Author Contributions: F.L., A.A.Z. and E.L. conceived of and designed the project. F.L., P.G.T. and B.R.M.N. performed the observations. F.L., E.L. and A.A. analysed the data. P.G.T., E.L. and J.K. contributed analysis tools. Q.A.P. contributed in the discussion. F.L. wrote the paper.

Funding: F.L. acknowledges support from the University of Hong Kong Postdoctoral Fellowships scheme and the Austrian Science Fund (AP23006, PI: Josef Hron). J.K. acknowledges support from the Philip Leverhulme Prize (PLP-2013-110, PI: Stefan Kraus) and from the research council of the KU Leuven under Grant Number C14/17/082.

Conflicts of Interest: The authors declare no conflict of interest.

\section{References}

1. Sánchez Contreras, C.; Sahai, R. OPACOS: OVRO Post-AGB CO (1-0) Emission Survey. I. Data and Derived Nebular Parameters. Astrophys. J. Suppl. Ser. 2012, 203, 16. [CrossRef]

2. Castro-Carrizo, A.; Quintana-Lacaci, G.; Neri, R.; Bujarrabal, V.; Schöier, F.L.; Winters, J.M.; Olofsson, H.; Lindqvist, M.; Alcolea, J.; Lucas, R.; et al. Mapping the ${ }^{12} \mathrm{CO} \mathrm{J}=1-0$ and J $=2-1$ emission in AGB and early post-AGB circumstellar envelopes. I. The COSAS program, first sample. Astron. Astrophys. 2010, 523, A59. [CrossRef]

3. Cox, N.L.J.; Kerschbaum, F.; van Marle, A.J.; Decin, L.; Ladjal, D.; Mayer, A.; Groenewegen, M.A.T.; van Eck, S.; Royer, P.; Ottensamer, R.; et al. A far-infrared survey of bow shocks and detached shells around AGB stars and red supergiants. Astron. Astrophys. 2012, 537, A35. [CrossRef]

4. Lagadec, E.; Verhoelst, T.; Mékarnia, D.; Suárez, O.; Zijlstra, A.A.; Bendjoya, P.; Szczerba, R.; Chesneau, O.; Van Winckel, H.; Barlow, M.J.; et al. A mid-infrared imaging catalogue of post-asymptotic giant branch stars. Mon. Not. R. Astron. Soc. 2011, 417, 32-92. [CrossRef] 
5. Lykou, F. Dusty Discs around Evolved Stars. Ph.D. Thesis, The University of Manchester, Manchester, UK, 2013.

6. Lykou, F.; Klotz, D.; Paladini, C.; Hron, J.; Zijlstra, A.A.; Kluska, J.; Norris, B.R.M.; Tuthill, P.G.; Ramstedt, S.; Lagadec, E.; et al. Dissecting the AGB star L2 Puppis: A torus in the making. Astron. Astrophys. 2015, 576, A46. [CrossRef]

7. Lykou, F.; Hron, J.; Zijlstra, A.A.; Tuthill, P.G.; Norris, B.R.M.; Kluska, J.; Paladini, C.; Lagadec, E.; Wittkowski, M.; Ramstedt, S.; et al. Unraveling disks in AGB stars. EAS Publ. Ser. 2015, 71-72, 217-222. [CrossRef]

8. Groenewegen, M.A.T.; Sevenster, M.; Spoon, H.W.W.; Perez, I. Millimetre observations of infrared carbon stars. II. Mass loss rates and expansion velocities. Astron. Astrophys. 2002, 390, 511. [CrossRef]

9. Miller Bertolami, M.M. New models for the evolution of post-asymptotic giant branch stars and central stars of planetary nebulae. Astron. Astrophys. 2016, 588, A25. [CrossRef]

10. Feast, M.W.; Whitelock, P.A.; Marang F. The case for asymmetric dust around a C-rich asymptotic giant branch star. Mon. Not. R. Astron. Soc. 2003, 346, 878-884. [CrossRef]

11. Lykou, F.; Zijlstra, A.A.; Kluska, J.; Lagadec, E.; Tuthill, P.G.; Avison, A.; Norris, B.R.; Parker, Q.A. The curious case of II Lup: A complex morphology revealed with SAM/NACO and ALMA. Mon. Not. R. Astron. Soc. 2018, 480, 1009. [CrossRef]

12. Lagage, P.O.; Pel, J.W.; Authier, M.; Belorgey, J.; Claret, A.; Doucet, C.; Dubreuil, D.; Durand, G.; Elswijk, E.; Girardot, P.; et al. Successful Commissioning of VISIR: The Mid-Infrared VLT Instrument. Messenger 2004, $117,12-16$.

13. Groenewegen, M.A.; Waelkens, C.; Barlow, M.J.; Kerschbaum, F.; Garcia-Lario, P.; Cernicharo, J.; Blommaert, J.A.D.L.; Bouwman, J.; Cohen, M.; Cox, N.; et al. MESS (Mass-loss of Evolved StarS), a Herschel key program. Astron. Astrophys. 2011, 526, A162. [CrossRef]

14. Rousset, G.; Lacombe, F.; Puget, P.; Hubin, N.N.; Gendron, E.; Fusco, T.; Arsenault, R.; Charton, J.; Feautrier, P.; Gigan, P.; et al. NAOS, the first AO system of the VLT: On-sky performance. Proc. SPIE 2003, 4839, 140-149. [CrossRef]

15. Lenzen, R.; Hartung, M.; Brandner, W.; Finger, G.; Hubin, N.N.; Lacombe, F.; Lagrange, A.M.; Lehnert, M.D.; Moorwood, A.F.; Mouillet, D. NAOS-CONICA first on sky results in a variety of observing modes. Proc. SPIE 2003, 4841, 944-952. [CrossRef]

16. Tuthill, P.G.; Monnier, J.D.; Danchi, W.C.; Wishnow, E.H.; Haniff, C.A. Michelson Interferometry with the Keck I Telescope. Publ. Astron. Soc. Pac. 2000, 112, 555-565. [CrossRef]

17. Tuthill, P.; Lacour, S.; Amico, P.; Ireland, M.; Norris, B.; Stewart, P.; Evans, T.; Kraus, A.; Lidman, C.; Pompei, E.; et al. Sparse aperture masking (SAM) at NAOS/CONICA on the VLT. Proc. SPIE 2010, 7735, 77351O. [CrossRef]

18. Thiébaut, E. MIRA: An effective imaging algorithm for optical interferometry. Proc. SPIE 2008, 7013, $7013 \mathrm{I}$. [CrossRef]

19. Kluska, J.; Malbet, F.; Berger, J.P.; Baron, F.; Lazareff, B.; Le Bouquin, J.B.; Monnier, J.D.; Soulez, F.; Thiébaut, E. SPARCO: A semi-parametric approach for image reconstruction of chromatic objects. Application to young stellar objects. Astron. Astrophys. 2014, 564, A80. [CrossRef]

20. De Beck, E.; Decin, L.; de Koter, A.; Justtanont, K.; Verhoelst, T.; Kemper, F.; Menten, K.M. Probing the mass-loss history of AGB and red supergiant stars from CO rotational line profiles. II. CO line survey of evolved stars: Derivation of mass-loss rate formulae. Astron. Astrophys. 2010, 523, A18. [CrossRef]

(C) 2018 by the authors. Licensee MDPI, Basel, Switzerland. This article is an open access article distributed under the terms and conditions of the Creative Commons Attribution (CC BY) license (http://creativecommons.org/licenses/by/4.0/). 